\title{
Hemangioma hepático poliquístico simulando un quiste hidatídico: Reporte de un caso y revisión del tema
}

Dres. Felipe Antonio Sánchez $T^{(1)}$, Nicolás Zugbe $G^{(1)}$, María Eugenia Lúcia $C^{(2)}$, Int. Marcela Moraga L(3).

1. Residente de Radiología, Universidad de los Andes. Santiago-Chile.

2. Radióloga, Servicio de Radiología, Clínica Santa María. Santiago-Chile.

3. Interna de Medicina, Universidad de Chile. Santiago-Chile.

\section{Polycystic liver hemangioma mimicking a hydatid cyst: A case report and review of the topic}

\begin{abstract}
Introduction: Cavernous hemangiomas are the most common benign mesenchymal hepatic tumor. Most have a typical appearance, but atypical forms have been reported that can make diagnosis difficult. Objective: To show a case of cystic hemangioma mimicking a hydatid cyst. Case: 60 year old woman with abdominal pain. Abdominal ultrasound: polycystic lesion with parietal calcifications, $6 \mathrm{~cm}$ in size, in the left hepatic lobe. Computed tomography of the abdomen shows the lesion as a simple cyst. During conversation the patient revealed she lives with 13 dogs. Hydatid disease was suspected and treatment with albendazole and segmental hepatectomy was indicated. A hemorrhagic tumor biopsy compatible with infarcted hemangioma was obtained. Discussion: The typical hepatic hemangioma is presented in ultrasound as an echogenic mass with posterior acoustic enhancement. The contrasted CT also presents with a classic pattern. Among the atypical presentations the cystic form is extremely rare and has only been mentioned in a few reports.
\end{abstract}

Keywords: Atypical hemangioma, Hemangioma cyst, Hydatid cyst.

Resumen: Introducción: Hemangioma cavernoso es el tumor hepático mesenquimático benigno más frecuente. La mayoría tiene un aspecto típico, pero se han reportado formas atípicas que pueden dificultar el diagnóstico. Objetivo: Mostrar un caso de hemangioma quístico simulando a un quiste hidatídico. Caso: Mujer de 60 años consulta por dolor abdominal. Ecografía abdominal: lesión poliquística con calcificaciones parietales de $6 \mathrm{~cm}$ en el lóbulo hepático izquierdo. Tomografía computada de abdomen muestra la lesión como un quiste simple. Dirigidamente la paciente revela convivir con 13 perros. Se sospechó hidatidosis y se indicó albendazol y hepatectomía segmentaria. Se obtuvo un tumor hemorrágico con biopsia compatible con hemangioma infartado. Discusión: El hemangioma hepático típico se presenta ecográficamente como una masa ecogénica con refuerzo acústico posterior. La TC contrastada también presenta un patrón clásico. Dentro de las presentaciones atípicas la forma quística es extremadamente rara y sólo ha sido mencionada en escasos reportes.

Palabras clave: Hemangioma atípico, Hemangioma quístico, Quiste hidatídico.

Sánchez F, Zugbe N, Lúcia ME, Moraga M. Hemangioma hepático poliquístico simulando un quiste hidatídico: Reporte de un caso y revisión del tema. Rev Chil Radiol 2014; 20(4): 164-167.

Correspondencia: Felipe Antonio Sánchez T. / fsancheztijmes@gmail.com

Trabajo recibido el 07 de noviembre de 2013. Aceptado para publicación el 26 de mayo de 2014.

\section{Introducción}

El hemangioma hepático cavernoso se define como una malformación vascular congénita y es considerado el tumor hepático benigno más frecuente ${ }^{(1)}$. Su prevalencia global varía entre un 1 a $2 \%$, siendo más frecuente en mujeres que hombres, con proporciones que varían de 2:1 a 5:1 dependiendo de las series analizadas ${ }^{(2)}$. La mayoría de los casos son asintomáticos y generalmente se detectan como un hallazgo incidental durante la ecografía abdominal de rutina ${ }^{(3)}$. Su tasa de complicaciones es baja (las cuales consisten principalmente en inflamación local, síndrome de Kassabach-Merrit, hemorragia o compresión de estructuras vecinas) y rara vez requieren 
de resolución quirúrgica(2). Dado todo lo anterior, es importante diferenciar al hemangioma cavernoso de otros tumores hepáticos que pudieran requerir un manejo más complejo. Es en este contexto donde los estudios imagenológicos aparecen como una herramienta esencial.

La mayoría de los hemangiomas hepáticos muestran un patrón ultrasonográfico característico, pero se ha reportado una amplia gama de presentaciones atípicas que pueden llevar a un error diagnóstico ${ }^{(2,3)}$. A continuación presentamos un caso con el fin de ampliar el espectro imagenológico del hemangioma cavernoso: un hemangioma hepático poliquístico simulando un quiste hidatídico. A nuestro conocimiento, existen sólo escasos reportes acerca de hemangiomas hepáticos quísticos descritos en la literatura médica ${ }^{(4-8)}$.

\section{Descripción del caso}

Paciente de sexo femenino de 60 años de edad, consulta por una historia de 3 meses de evolución caracterizada por dolor moderado en hemiabdomen superior, plenitud postprandial, meteorismo y anorexia. Dentro de sus antecedentes médicos destaca hipertensión arterial, dislipidemia y tabaquismo. Además, reside en una zona rural y convive con aproximadamente 13 perros. Al examen físico no se encontraron hallazgos significativos.

Se inició el estudio con ecografía abdominal que reveló la presencia de una gran imagen quística multilobulada de $6.1 \mathrm{~cm}$ de diámetro en el lóbulo hepático izquierdo, con imágenes hiperecogénicas en su pared sugerentes de calcificaciones parietales (Figura 1). La lesión desplazaba parcialmente la vena suprahepática media y al Doppler no mostraba estructuras vasculares (Figura 2). Concomitantemente se observó colecistolitiasis. El resto del examen fue normal.

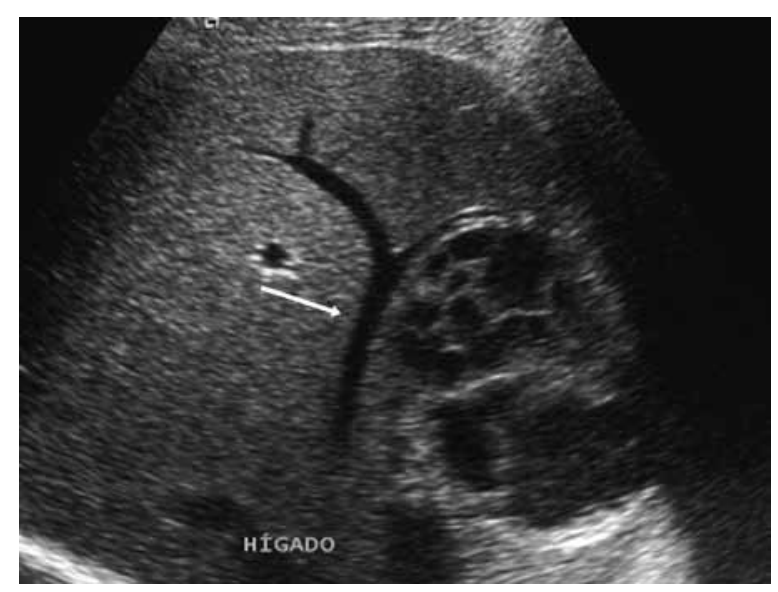

Figura 1. Sección axial de ecotomografía de abdomen a nivel del hígado muestra una lesión quística tabicada en el lóbulo hepático izquierdo adyacente a la vena hepática media (flecha) con los caracteres de un quiste hidatídico.

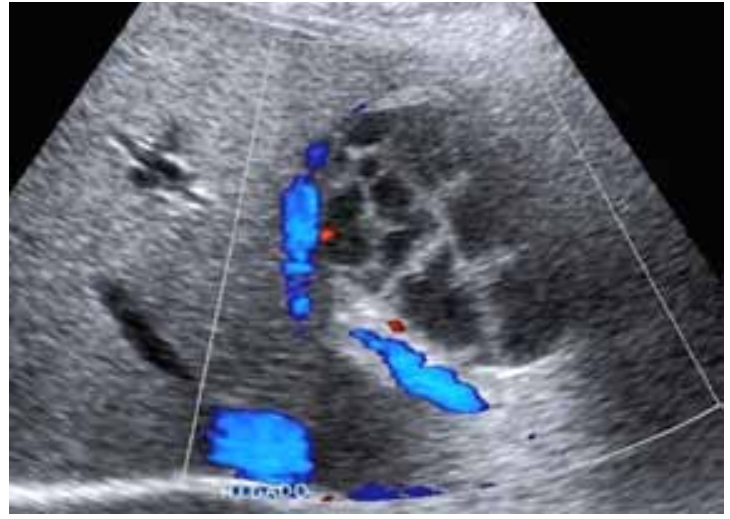

Figura 2. Al estudiar la lesión con Doppler color se evidencia que la lesión no presenta flujo vascular significativo y que respeta a la vena hepática vecina.

Estos hallazgos sugerían fuertemente hidatidosis como el diagnóstico principal, basado en las características imagenológicas típicas del quiste hidatídico (ver discusión). Se solicitó mayor estudio: la tomografía axial computada (TAC) de abdomen exhibía la misma lesión como un quiste homogéneo (a diferencia del ultrasonido) con calcificaciones parietales, ubicado en los segmentos hepáticos III y IV b (Figuras 3 y 4). La TAC de tórax no mostró compromiso torácico.

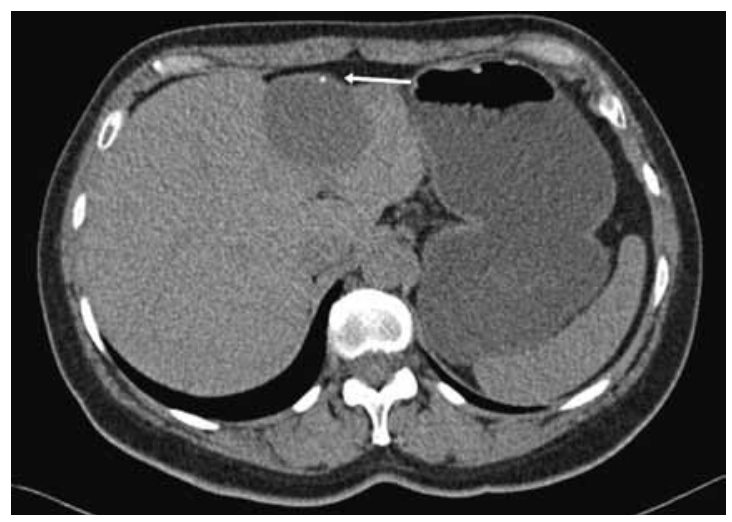

Figura 3. Tomografía computada de abdomen no contrastada que evidencia lesión de densidad quística con calcificación puntiforme en su pared (Flecha). Este estudio no muestra la naturaleza tabicada de la lesión, a diferencia del ultrasonido.

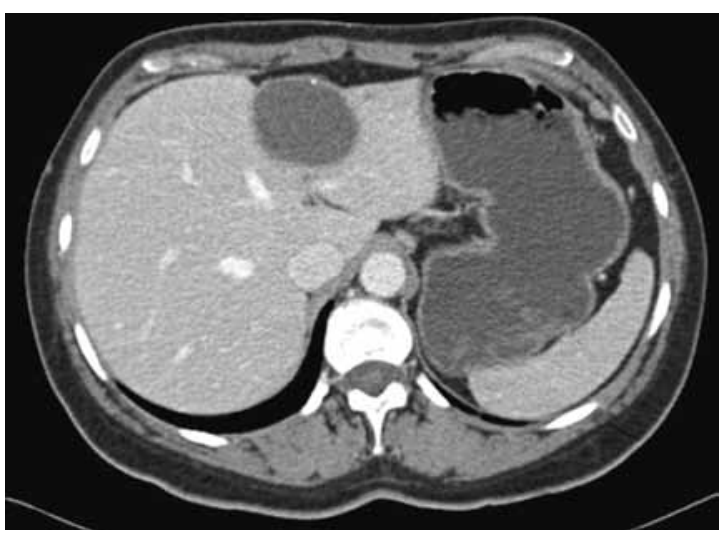

Figura 4. Al inyectar contraste, la lesión no presenta realce periférico ni de sus tabiques. 
De acorde a lo anterior, se indicó tratamiento con Albendazol y de manera diferida se realizó una hepatectomía segmentaria más colecistectomía. Se envió a biopsia un fragmento de $3 \times 4 \times 3 \mathrm{~cm}$ el cual contenía un tumor bien definido, de aspecto hemorrágico. La biopsia final informó: "Formación fibrosa con necrosis central y depósitos de fibrina, consistente con hemangioma infartado" (Figuras 5 y 6 ).

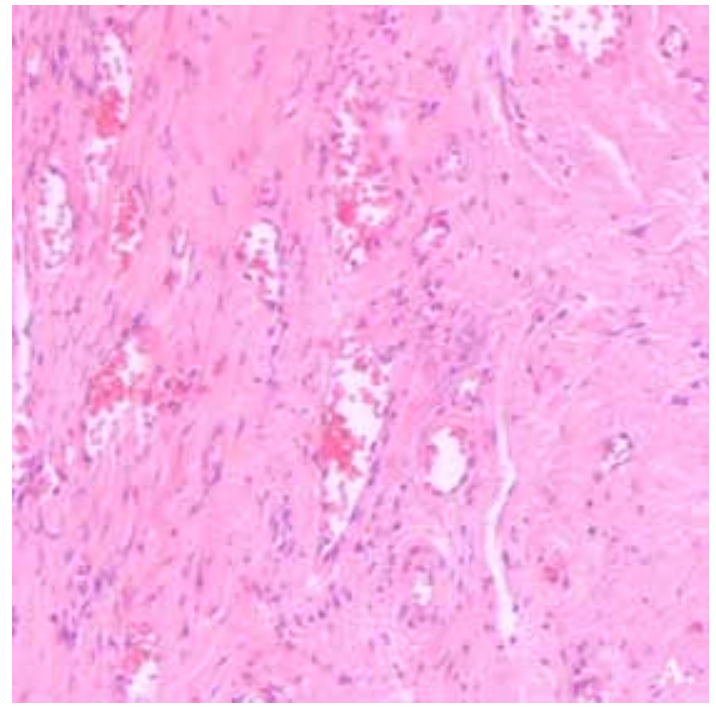

Figura 5. Microfotografía 100x, tinción Hematoxilina-Eosina (Cortesía Dr. Manuel Meneses). Los cortes patológicos muestran vasos sanguíneos de disposición lacunar embebidos en una matriz de tejido conectivo, características típicas de un hemangioma.

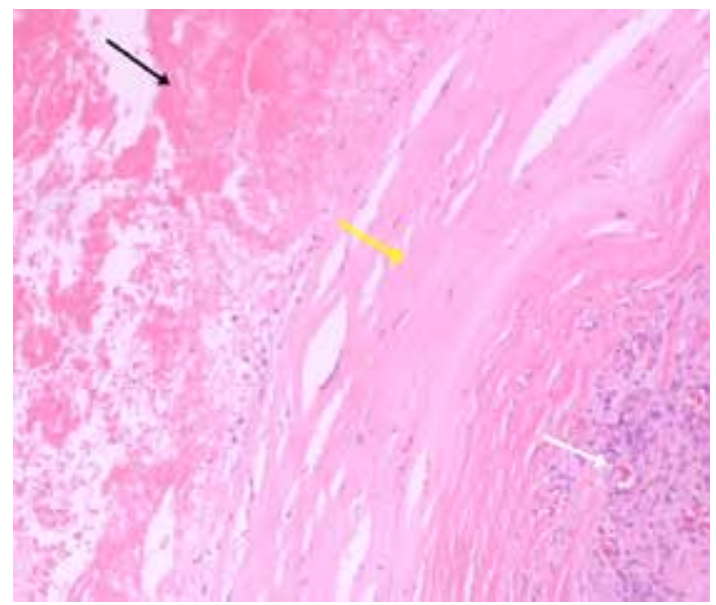

Figura 6. La lesión mostró extensas áreas de infarto (flecha blanca), se observa también el tabique compuesto de tejido conectivo (flecha negra) y el área respetada del hemangioma (flecha amarilla).

\section{Discusión}

La representación ecográfica clásica del hemangioma hepático es una masa hiperecogénica, homogénea y bien definida, con refuerzo acústico posterior (Figuras 7 y 8 ). Los hallazgos típicamente descritos en la TAC consisten en una lesión hipodensa en los cortes sin contraste. En cortes contrastados, la fase arterial muestra captación de contraste precoz en la región periférica del tumor, siguiendo un patrón globular $^{(2)}$. En la fase venosa se observa un realce centrípeto que progresa a un llenado uniforme de la lesión; dicho patrón persiste en cortes tardíos ${ }^{(2)}$.

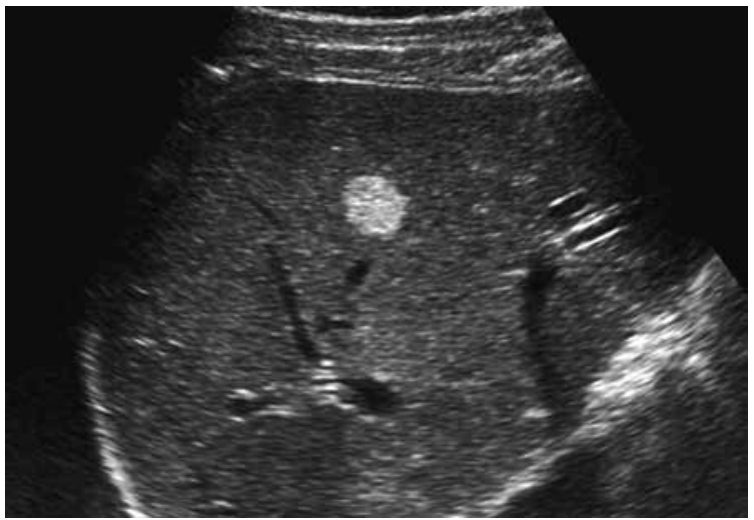

Figura 7. Aspecto ecográfico de un hemangioma hepático típico. La lesión es bien definida, ecogénica, con refuerzo acústico posterior.

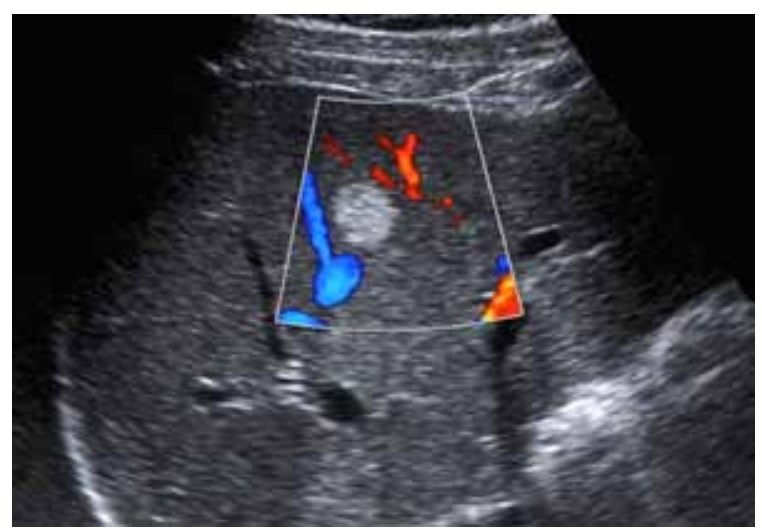

Figura 8. Al estudiar la lesión con Doppler color no se observa flujo vascular.

Se han descrito muchas presentaciones imagenológicas atípicas, pero entre ellas la forma quística destaca como un hallazgo extremadamente raro, sólo con algunos pocos casos reportados en la literatura $^{(2)}$. Tres de éstos se han presentado como un quiste hepático complejo diagnosticado de manera incidental en una paciente de características similares $^{(4,7,8)}$. Otro reporte describe un hemangioma hepático manifestado como un quiste hepático gigante uniloculado que clínicamente debutó como un síndrome de Budd-Chiari(5).

Este caso se presentó como un desafío diagnóstico debido a que la historia clínica y los hallazgos imagenológicos eran consistentes con el diagnóstico de hidatidosis. La ubicación más común de los 
quistes hidatídicos es el hígado (75\%), seguido de los pulmones (15\%) y otras regiones anatómicas $(10 \%)^{(7)}$. Los quistes hidatídicos se muestran en la ecografía como formaciones quísticas que pueden ser simples o multiloculadas dependiendo de la presencia de vesículas hijas. Tienen un contorno hiperecogénico bien definido y exhiben una sombra acústica posterior. Sus paredes son representadas como dos líneas ecogénicas separadas por una capa hipoecogénica y con frecuencia se reportan calcificaciones parietales. Los quistes simples no muestran estructuras internas, sin embargo en ocasiones se pueden ver membranas flotantes o múltiples focos ecogénicos que corresponden a arena hidatídica en su interior ${ }^{(7)}$. La TAC muestra los mismos hallazgos que el ultrasonido: una lesión quística hipodensa y con paredes de densidad aumentada en cortes no contrastados $^{(7)}$.

Vale mencionar el valor del ultrasonido, en este caso para determinar la arquitectura interna de la lesión estudiada, esto debido a la gran resolución espacial que posee ${ }^{(8)}$. La TAC, a diferencia del ultrasonido, tiene limitaciones para definir las estructuras internas de un quiste, como se observa en este caso al no mostrar los septos internos de éste.

A modo de conclusión, a pesar de que es infrecuente, la posibilidad de un hemangioma quístico podría ser incluida en el diagnóstico diferencial de un quiste hepático, una vez que los diagnósticos más comunes se hayan descartado.

\section{Agradecimientos}

Agradecimiento al Dr. Manuel Meneses por facilitar las microfotografías.

\section{Bibliografía}

1. Yoon S, Charny C, Fong Y, Jarnagin W, Schwartz L, Blumgart L. Diagnosis, management, and outcomes of 115 patients with hepatic hemangioma. J Am Coll Surg 2003; 197: 392-402.

2. Vilgrain V, Boulos L, Vullierme M, Denys A, Terris B, Menu Y. Imaging of atypical hemangiomas of the liver with pathologic correlation. Radiographics 2000; 20: 379-397.

3. Kim K, Kim T, Han J, Kim A, Lee H, Park S, et al. Hepatic hemangiomas: spectrum of US appearances on gray-scale, power poppler, and contrast-enhanced US. Korean J Radiol 2000; 1: 191-197.

4. Cha E, Kim K, Choi Y, Song J, Cho K, Lee M. Multicystic cavernous haemangioma of the liver: ultrasonography, CT, MR appearances and pathological correlation. $\mathrm{Br}$ J Radiol 2008; 81: e37-39.

5. Hanazaki K, Koide N, Kajikawa S, Ushiyama T, Watanabe T, Adachi W, et al. Cavernous hemangioma of the liver with giant cyst formation: degeneration by apoptosis? J Gastroenterol Hepatol 2001; 16(3): 352-355.

6. Hihara T, Araki T, Katou K, Odashima H, Ounishi H, Kachi K, et al. Cystic cavernous hemangioma of the liver. Gastrointest Radiol 1990; 15(2): 112-114.

7. Nakachi A, Shiraishi M, Shimoji H, Tomori T, Oshiro $\mathrm{T}$, Muto Y. Multicystic cavernous hemangioma of the liver: report of a case including diagnostic imaging and pathologic correlation. Radiat Med 1998; 16(3): 209-212.

8. Hussain M, Ohtomo K, Hihara T, Uchiyama G, Ainoda T, Yamamoto M, et al. Multilocular cystic hemangioma: CT and MRI appearance. Radiat Med 1992; 10(5): 206209.

9. Pedrosa I, Saíz A, Arrazola J, Ferreirós J, Pedrosa C. Hydatid disease: radiologic and pathologic features and complications. Radiographics 2000; 20(3): 795-817.

10. Hangiandreou N. AAPM/RSNA physics tutorial for residents. Topics in US: B-mode US: basic concepts and new technology. Radiographics 2003; 23(4): 1019-1033. 